SHORT REPORT

\title{
Simple and safe treatment of pretibial haematoma in elderly patients
}

\author{
G S Karthikeyan, S Vadodaria, P R W Stanley
}

Emerg Med J 2004;21:69-70

A simple and safe technique is described for evacuation of pretibial haematoma in elderly patients. A Yankauer sucker attached to the wall suction is used to evacuate the haematoma under local anaesthesia. This technique is used in the accident and emergency department and the ward.

$\mathrm{T}$ reatment of lower limb haematomas in elderly patients is complex. These patients often have multiple medical problems such as respiratory problems, cardiac problems, and arthritis. They are often taking drugs including warfarin, corticosteroids, and aspirin. Conventionally, patients with pretibial haematomas are managed under general or regional anaesthesia for evacuation of the haematoma and skin grafting if required. ${ }^{1}$ While the patient on the ward, waiting for results of investigations and optimising the patients general condition for surgery, the skin over the haematoma may undergo vascular compromise leading to skin necrosis and subsequent infection. The time taken for these patients to be assessed as fit for general or regional anaesthesia and for their overall condition to be optimised can compound the problem with necrosis of the overlying skin, contamination, and infection. ${ }^{2}$ The time spent may delay evacuation of the haematoma to the point where total skin loss over the

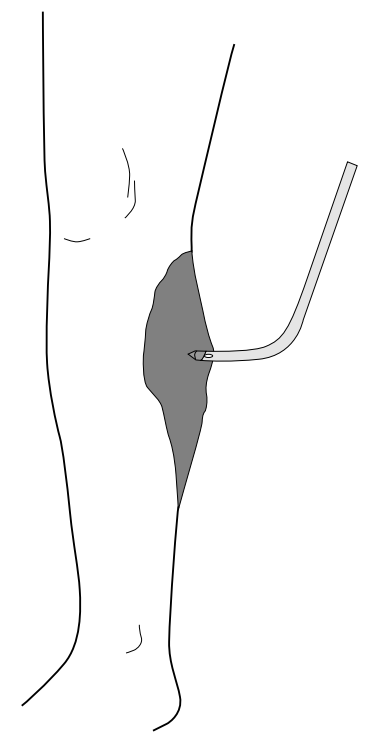

B

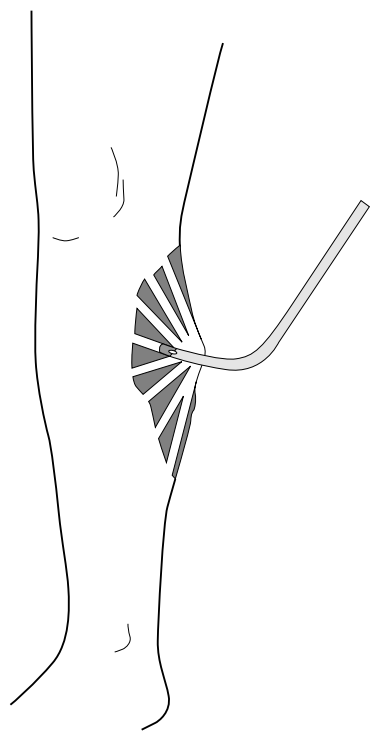

Figure 1 (A) A small incision is made on the overlying skin and the suction cannula is inserted into the haematoma. (B) The clot is broken into small pieces by to and fro movement of the cannula as in liposuction. haematoma results, necessitating skin grafts to the traumatised area.

Postoperatively these patients are at risk of developing deep vein thrombosis, pulmonary embolism, and other cardiovascular complications because of immobilisation. ${ }^{34}$

We evacuate these pretibial haematoma as soon as possible after the injury, to reduce the risks of skin necrosis and secondary infection. The procedure can be done without the need for general anaesthesia and postoperative immobilisation.

A safe and simple technique is described in this paper, which is used in the accident and emergency department or on the wards under local anaesthesia.

\section{TECHNIQUE}

\section{Position and pain control}

The procedure is performed with the injured leg elevated. The anaesthesia can be infiltration of local anaesthetic, haematoma block using bupivacaine with adrenaline and hylase or entonox (depending upon the availability).

\section{Inncision (fig 1A)}

If there is a laceration along with the haematoma, this laceration is used as a port for the sucker or a stab incision is made in the skin overlying the haematoma.

\section{Evacuation (fig 1B)}

The Yankauer suction cannula is attached to the wall suction. The haematoma is then evacuated by to and fro movement of the cannula in the haematoma, which breaks the clots and sucks it as in liposuction. The cavity is then irrigated with normal saline. The laceration or the incision is left open for free drainage or can be closed with adhesive tapes (figs 2 and 3). ${ }^{5}$ The leg is dressed with compression dressing (fig 4). The patient is mobilised after the procedure (fig 5).

\section{DISCUSSION}

This technique allows us to evacuate the haematoma as soon as possible without general anaesthesia in the accident and emergency department or on the wards. The early evacuation of the haematoma avoids pressure necrosis of the overlying

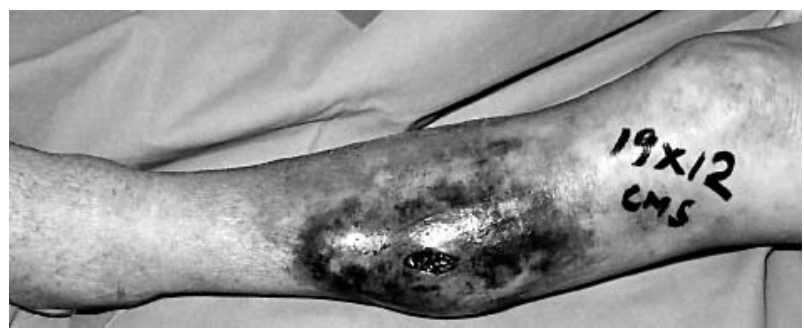

Figure 2 Large traumatic haematoma of the leg. The patient had associated heart failure. 


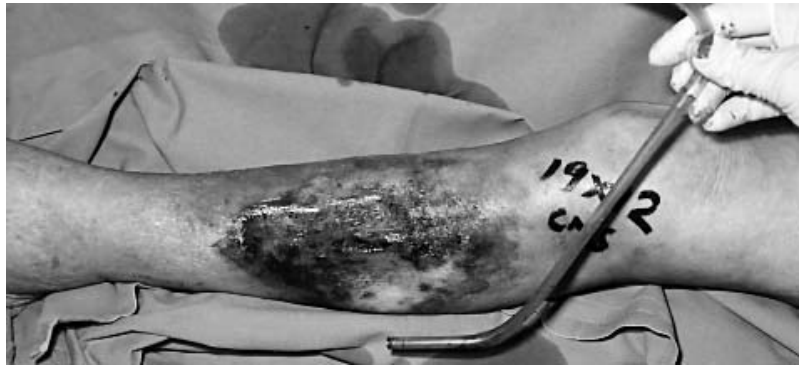

Figure 3 The haematoma was drained using Yankauer suction cannula and a wall suction.

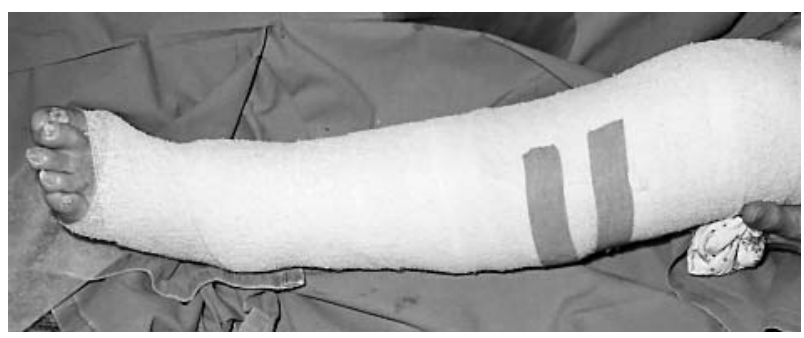

Figure 4 Gentle compression dressing applied.

skin and secondary infection. It also helps us to buy some time to investigate the patient for fitness for general anaesthesia if a skin graft is required. Hence we can convert an emergency operation into an urgent elective operation. The technique is versatile, as it requires few instruments, the local anaesthetic agent, Yankauer suction cannula, wall or portable suction and compression dressings, which are readily available in accident and emergency departments and in wards.

The Yankauer suction cannula is a very efficient suction cannula with blunt end, multiple fenestrations and is available in sterilised form in different lengths. Compression

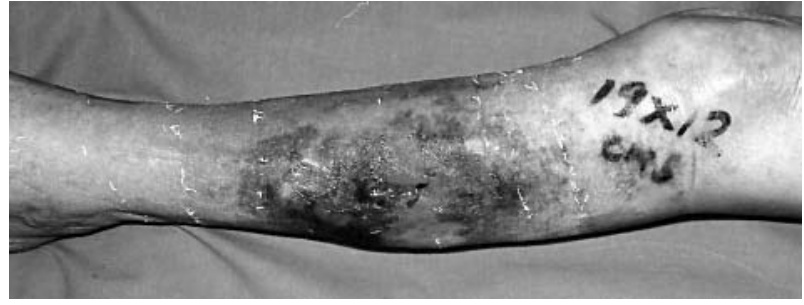

Figure 5 There is no further collection of the blood and the wound is healing in progress without any infection one week after the procedure.

bandaging is important to prevent further collection of haematoma and it also facilitates firm contact between degloved skin and underlying soft tissue.

It is not necessary to immobilise the patient and in appropriate cases may not require hospitalisation and can be treated as outpatient basis for change of dressing and monitoring wound healing.

\section{Authors' affiliations}

G S Karthikeyan, S Vadodaria, P R W Stanley, Department of Plastic and Reconstructive Surgery, Castle Hill Hospital, Hull, UK

Correspondence to: Mr S Vadodaria, 24 Gisborne Road, Sheffield S1 1 7HB, UK; bvadodaria@aol.com

Accepted for publication 14 April 2003

\section{REFERENCES}

1 Tandon SN, Sutherland AB. Pretibial Lacerations. Br J Plast Surg 1973;26:192.

2 Warren RA, Warren MA, Buswell WA, et al. Wound healing and infection in pretibial lacerations. Ann Plast Surg 1991;26:243-7.

3 Luscombe JC. Mobilisation after skin grafting of pretibial lacerations. Br J Plast Surg 2001:54:646-7.

4 Crawford BS, Gipson M. The conservative management of pretibial lacerations in elderly patients. Br J Plast Surg 1977;30:174-6.

5 Sutton R, Pritty P. Use of sutures or adhesive tapes for primary closure of pretibial lacerations. BMJ 1985;290:1627. 